\title{
Decoherence of Black Holes by Hawking Radiation
}

\author{
JEAN-GUY DEMERS \\ Department of Physics, McGill University \\ Ernest Rutherford Building, 3600 University Street \\ Montréal, PQ, Canada H3A 2T8 \\ Claus Kiefer \\ Fakultät für Physik, Universität Freiburg \\ Hermann - Herder - Strasse 3 \\ D-79104 Freiburg, Germany
}

\begin{abstract}
We discuss in detail the semiclassical approximation for the CGHS model of two-dimensional dilatonic black holes. This is achieved by a formal expansion of the full Wheeler-DeWitt equation and the momentum constraint in powers of the gravitational constant. In highest order, the classical CGHS solution is recovered. The next order yields a functional Schrödinger equation for quantum fields propagating on this background. We show explicitly how the Hawking radiation is recovered from this equation. Although described by a pure quantum state, the expectation value of the number operator exhibits a Planckian distribution with respect to the Hawking temperature. We then show how this Hawking radiation can lead to the decoherence of black hole superpositions. The cases of a superposition of a black hole with a white hole, as well as of a black hole with no hole, are treated explicitly.
\end{abstract}

McGill 95-56

Freiburg THEP-95/22 


\section{Introduction}

One of the main applications of any quantum theory of gravity should be the complete understanding of black hole evaporation. Since such a theory is not yet available, attention has been focused on simpler models whose quantization is expected to be tractable. Most notably among these are the models of two-dimensional dilaton gravity (see, for example, [1] for a detailed review). Although much insight has been gained into the evaporation process and, in particular, the back reaction of the Hawking radiation onto the black hole and the problem of "information loss", a full understanding of the complete, non-perturbative, evolution remains elusive. The reason for this is that even for such models the full quantum theory is not known.

At present, the most popular approaches towards the quantization of gravity are superstrings and quantum general relativity. While many of the models discussed are in fact "string-inspired", a non-perturbative understanding of the full string field theory is not in sight. Effort is therefore also concentrated on a more conservative approach: The application of canonical quantization rules to the general theory of relativity. While this fails to yield, in four spacetime dimensions, a viable theory in a perturbative sense, it might nevertheless make sense non-perturbatively. Even if this were not the case, this framework appears appropriate to exhibit the main physical problems and to suggest methods for their solution. We shall thus restrict our investigation to this framework, too.

What progress has been made towards a quantum theory of black holes within canonical quantum gravity? The situation of a spherically symmetric black hole without matter has been completely solved [2, 3]. After the embedding variables describing the location of three-dimensional hypersurfaces in spacetime are isolated, the remaining state is a quantum mechanical wave function, $\psi(M)$, where $M$ is the mass of the hole. The variable conjugate to $M$ is related to the time in the asymptotic region. The fact that no field theoretic degrees of freedom are present reflects of course the situation of the classical theory (Birkhoff's theorem). In more complicated models where matter degrees of freedom are present it seems no longer possible to proceed as in [2, 3] and to isolate the embedding variables from the "true" degrees of freedom [i]. We shall thus stick to the approach where all degrees of freedom are treated on an equal footing as dynamical variables.

Instead of finding the full solution in simple situations, approximation 
schemes have been employed in the full theory. One example is a formal expansion of the full wave functional in powers of the gravitational constant $G$ [5]. The $G$-expansion scheme allows one to derive the limit of quantum field theory in a background spacetime as well as to find quantum gravitational correction terms to this limit.

One application of such correction terms for black holes was made in [6] where it was shown that some of these terms yield non-unitary contributions which become relevant if the black hole mass approaches the Planck mass. This could be of central importance for the information loss problem. The investigation in [6] was, however, only heuristic, since it did not pay attention to problems of regularization. One of the motivations for the present paper is the attempt to study the semiclassical expansion in the much simpler context of two-dimensional dilatonic gravity. This will enable us, in particular, to understand the emergence of Hawking radiation in this framework, which thus complements the standard derivation with the use of Bogolubov coefficients.

Furthermore, it will also be straightforward to study the validity of the limit where spacetime is semiclassical and matter fully quantum. This question received some interest recently because it was found in [7] that semiclassical gravity breaks down on hypersurfaces which capture both the infalling matter near the horizon and the Hawking radiation. In our paper we shall consider superpositions of different semiclassical components and investigate to which extent they become dynamically independent. The key ingredient is thereby played by the notion of decoherence - the emergence of quasiclassical properties through the irreversible interaction with the environment (see, for example, [5] and the references therein). What can possibly play the role of the environment for the black hole? It is the Hawking radiation itself, which may serve as the decohering agent. Although it may be weak, it possesses a large entropy capacity due to its many degrees of freedom and can thus carry all the information about the superpositions which thereby decohere.

Our paper is organized as follows. In Section 2 we shall review the canonical formalism for the CGHS model of dilatonic gravity, in particular the corresponding Wheeler-DeWitt equation and the quantum momentum constraint. Section 3 is then devoted to a detailed discussion of the semiclassical approximation. The highest order yields the Hamilton-Jacobi equation for dilatonic gravity without matter fields. We show how the classical CGHS solution can be recovered from a solution to this equation. We also comment on a connection between this solution and the entropy of the black hole. We 
then derive in Section 4 the functional Schrödinger equation for matter fields on the semiclassical background. An important part of our paper is then devoted to a derivation of Hawking radiation from this Schrödinger equation. We consider both the case of a collapsing geometry and of an eternal black hole. We shall find that Hawking radiation is obtained from a pure quantum state in the sense that the expectation value of the number operator for field quanta exhibits a thermal spectrum with respect to the Hawking temperature. We end this section with a brief discussion of quantum gravitational correction terms to the functional Schrödinger equation. In Section 5 we discuss in detail how Hawking radiation can lead to decoherence for superpositions of black hole states. In particular, we study the superposition of a black hole with a white hole as well as the superposition of a black hole with no hole. The results then justify the separate consideration of each semiclassical component, since they dynamically decouple from each other. Finally, Section 6 contains a brief conclusion as well as an outlook on future work.

\section{Quantum theory of two-dimensional black holes}

In this section we shall apply canonical quantum gravity to spherically symmetric black holes. From the technical point of view, this lies between the full quantum theory and finite-dimensional minisuperspace models, since the wave functional depends now on one-dimensional fields. An effective twodimensional theory can thus be obtained by imposing a spherical symmetric ansatz for the classical four-dimensional metric and applying the standard ADM procedure for the remaining degrees of freedom.

Recently, however, a slightly modified two-dimensional theory has received considerable attention [8]. Apart from being "string inspired", the reason for the choice of this "CGHS model" is basically simplicity, giving rise to the hope that the full quantum evolution (including the back reaction of Hawking radiation on the gravitational background) can be tackled in this framework. For this reason we restrict our attention to this model, too, but keep in mind that this will give at best some hints of how to proceed in the full theory. 
The action for the two-dimensional gravity model of CGHS coupled to $N$ massless scalar fields reads

$$
S=\frac{1}{G} \int d x d t \sqrt{-\bar{g}} e^{-2 \bar{\phi}}\left(\bar{R}+4(\bar{\nabla} \bar{\phi})^{2}+4 \lambda^{2}\right)-\frac{1}{2} \int d x d t \sqrt{-\bar{g}} \sum_{i=1}^{N}\left(\bar{\nabla} f_{i}\right)^{2}
$$

where $\bar{\phi}$ is the dilaton field, $f_{i}$ are the matter fields and $G$ is the (unitless) gravitational coupling constant, which we have introduced for later convenience. We note that in the corresponding model resulting from dimensional reduction the term $4 \lambda^{2}$ is replaced by $e^{2 \bar{\phi}} / l^{2}$, where $l^{2}$ is a relic of the curvature components orthogonal to the two-sphere, which usually is measured in terms of the Planck length. In this sense the two-dimensional "cosmological constant term" is an artefact which has its origin in the "real" (four-dimensional) gravitational constant. Alternatively, $\lambda^{-1}$ may be viewed as being related to the magnetic charge of four-dimensional dilatonic black holes.

In order to study the canonical structure of (1), we make the transformation $\phi=e^{-2 \bar{\phi}}$ and $g_{\alpha \beta}=e^{-2 \bar{\phi}} \bar{g}_{\alpha \beta}$, which eliminates the kinetic term for the dilaton. This yields

$$
S=\int d x d t \sqrt{-g}\left[\frac{1}{G}\left(R \phi+4 \lambda^{2}\right)-\frac{1}{2}(\nabla f)^{2}\right],
$$

where we have written out only one of the scalar fields for simplicity. Following [9] we write the metric in an ADM-like parametrization:

$$
d s^{2}=e^{2 \rho}\left[-\sigma^{2} d t^{2}+(d x+\xi d t)^{2}\right],
$$

where $\sigma$ denotes the lapse and $\xi$ the shift function (note the additional factor $e^{2 \rho}$ in the definition of the metric, which differs from the standard ADM convention). The action then reads, upon neglecting surface terms ${ }^{1}$

$$
S=\int d x d t\left(\dot{\phi} \Pi_{\phi}+\dot{\rho} \Pi_{\rho}+\dot{f} \Pi_{f}-\xi \mathcal{F}-\sigma \mathcal{G}\right),
$$

where

$$
\Pi_{\phi}=\frac{2}{\sigma G}\left(\xi \rho^{\prime}+\xi^{\prime}-\dot{\rho}\right)
$$

\footnotetext{
${ }^{1}$ The surface term describing the ADM energy, which is required in the Hamiltonian in order to consistently recover Hamilton's equations of motion, is not needed for the constraint analysis below and can thus consistently be neglected at this stage.
} 


$$
\begin{aligned}
\Pi_{\rho} & =\frac{2}{\sigma G}\left(\xi \phi^{\prime}-\dot{\phi}\right), \\
\Pi_{\sigma} & \approx 0, \quad \Pi_{\xi} \approx 0, \\
\Pi_{f} & =\frac{1}{\sigma}\left(\dot{f}-\xi \phi^{\prime}\right) .
\end{aligned}
$$

Here, primes and dots respectively denote a derivative with respect to $x$ and $t$, while " $\approx 0$ " is used to specify the location of the constraint surface in phase space. The momentum and Hamilton ian constraints are then given by the expressions

$$
\begin{aligned}
\mathcal{F} & =\rho^{\prime} \Pi_{\rho}-\Pi_{\rho}^{\prime}+\phi^{\prime} \Pi_{\phi}+\phi^{\prime} \Pi_{f} \approx 0, \\
\mathcal{G} & =\frac{2}{G} V_{G}-\frac{G}{2} \Pi_{\phi} \Pi_{\rho}+\frac{1}{2} \Pi_{f}^{2}+V_{M} \approx 0 .
\end{aligned}
$$

where

$$
V_{G}=4\left(\phi^{\prime \prime}-\phi^{\prime} \rho^{\prime}-2 \lambda^{2} e^{2 \rho}\right), V_{M}=\frac{1}{2} f^{\prime 2} .
$$

The fields $\rho$ and $\phi$ don't enter the momentum constraint on the same footing, since the former transforms as a density, whereas the latter transforms as a scalar. Quantization then proceeds à la Dirac by imposing these constraints as restrictions on physically allowed wave functionals $\Psi[\rho(x), \phi(x), f(x)]$ and assuming the usual equal-time commutation relations, $\left[\rho(x), \Pi_{\rho}(y)\right]=i \delta(x-$ $y$ ) etc., are obeyed. Note that this may not be consistent, since $\phi$ is restricted, by the above redefinition, to positive values. Affine commutation relations would thus be more appropriate. However, we do not expect this difference to be relevant for the present discussion which focuses on a semiclassical expansion. Note also that this quantization procedure differs from methods recently employed in this context [2], where the constraints are first manipulated on the classical level to isolate explicitly the embedding variables (which have to be spacetime scalars), with respect to which a Schrödinger equation is then obtained after quantization.

Since the supermetric is flat in these field variables, there is no factor ordering ambiguity in the Hamiltonian constraint, and one readily obtains

$$
\begin{aligned}
\mathcal{H}_{\|} \Psi & =\left(\rho^{\prime} \frac{\delta}{\delta \rho}-\frac{d}{d x} \frac{\delta}{\delta \rho}+\phi^{\prime} \frac{\delta}{\delta \phi}+f^{\prime} \frac{\delta}{\delta f}\right) \Psi=0 \\
\mathcal{H}_{\perp} \Psi & =\left(\frac{G}{2} \frac{\delta^{2}}{\delta \rho \delta \phi}-\frac{1}{2} \frac{\delta^{2}}{\delta f^{2}}+\frac{1}{2 G} V_{G}+V_{M}\right) \Psi=0 .
\end{aligned}
$$


On a first glance, (9) seems to describe an almost trivial field theory, the only "interaction" coming from the Liouville term proportional to $e^{2 \rho}$. In particular, the field $f$ has totally decoupled from the gravitational fields $\rho$ and $\phi$, a consequence of the conformal coupling in two dimensions. However, the presence of the momentum constraints induces a "correlation interaction" [10] which prevents the formulation of solutions separating in the respective variables. A major problem also seems to be the occurrence of anomalies which spoil the validity of (8) and (9) unless a quantum modification of the Hamiltonian is made [10]. Since this may be of minor relevance for the topic of this paper, these problems will not be discussed here. We hope, however, to return to these issues in a future publication.

\section{The semiclassical expansion}

Since it is not yet clear how the full equations (8) and (9) can be addressed rigorously, we turn here to a semiclassical expansion scheme. Such a scheme is also interesting on its own, as Hawking radiation can be properly understood in that context. This seems indeed appropriate as long as one focuses on the evolution of quantum fields on a semiclassical gravitational background [5]. More precisely, this expansion is of the Born-Oppenheimer type with respect to the gravitational constant. It is for this purpose that we have introduced the dimensionless constant $G$ in (11). Alternatively, one can perform this expansion with respect to the - large - number $N$ of matter fields, where one starts from a Liouville-type action in which a nonlocal redefinition of the fields in (11 has been preformed [11]. The appropriate parameter there turns out to be $\kappa \equiv(N-24) / 6$. Since in [11], $\kappa$ takes the same place in the Hamiltonian constraint that in our case is taken by $G$, both schemes are formally equivalent. We choose to perform an expansion with respect to $G$. We note, however, that this scheme is different from the one where the semiclassical Einstein equations (with the expectation value of the energymomentum tensor on the right-hand side) are discussed in the limit of large $N$ [1]. While this can serve to suppress graviton loops compared to matter loops, the present approximation scheme takes also into account quantum gravitational correction terms, see Section 4.4 .

We thus assume an ansatz of the form

$$
\Psi[\rho, \phi, f]=e^{i\left(G^{-1} S_{0}+S_{1}+G S_{2}+\ldots\right)} .
$$


At order $G^{-2}$ one finds $\delta S_{0} / \delta f=0$, while at order $G^{-1}$ we obtain:

$$
\frac{\delta S_{0}}{\delta \phi} \frac{\delta S_{0}}{\delta \rho}=4\left(\phi^{\prime \prime}-\phi^{\prime} \rho^{\prime}-2 \lambda^{2} e^{2 \rho}\right)=V_{G}
$$

which is the Hamilton- Jacobi equation for pure gravity. The solution of (11) was found in [9] to be

$$
S_{0}=\int d x\left[Q+\phi^{\prime} \ln \left(\frac{2 \phi^{\prime}-Q}{2 \phi^{\prime}+Q}\right)\right]
$$

with $Q=2 \sqrt{{\phi^{\prime}}^{2}+\left(C-4 \lambda^{2} \phi\right) e^{2 \rho}}$, where $C$ is an integration constant. In fact, $C$ is a constant for the full set of constraints in the pure gravity case, since it can be shown [9] that the spatial derivative of the functional

$$
C\left[\rho, \Pi_{\rho}, \phi\right] \equiv e^{-2 \rho}\left(\frac{\Pi_{\rho}^{2}}{4}-\phi^{\prime 2}\right)+4 \lambda^{2} \phi
$$

is equal to a linear combination of the constraints. Note that Eq.(12) obeys $\delta S_{0} / \delta \phi=V_{G} / Q$ and $\delta S_{0} / \delta \rho=Q$. Moreover, $C$ commutes with all constraints and can thus be interpreted as an "observable" - in fact, it is proportional to the ADM mass (see below). An analogous quantity appears in the reduced models from four dimensions [2, 3]. In the presence of matter fields, the spatial derivative of the functional $C$ no longer vanishes on the constraint surface. Therefore, the general approach to reduction made in [2, 3] is no longer applicable here, see [4].

It is also easily checked that $S_{0}$ obeys the momentum constraint in this order of approximation (since this constraint does not contain $G$, its expansion is straightforward). Note that $S_{0}$ becomes imaginary if either $Q^{2}<0$ or $4 \phi^{\prime 2}-Q^{2}<0 \Leftrightarrow C>4 \lambda^{2} \phi$ (both conditions cannot be satisfied simultaneously). For the black hole solution discussed below, the latter condition describes the region inside the horizon.

\subsection{Spacetime and dilaton}

We now recover explicitly the classical solutions for the conformal factor of the metric as well as for the dilaton, $\rho(x, t)$ and $\phi(x, t)$, from the solution 
(12) to the Hamilton-Jacobi equation. The momenta are given in (5), and using $\Pi_{\theta}=G^{-1} \delta S_{0} / \delta \theta$ for $\theta=\rho$ and $\phi$ one has

$$
\begin{aligned}
\frac{2}{\sigma}\left(\xi \rho^{\prime}+\xi^{\prime}-\dot{\rho}\right) & =\frac{\delta S_{0}}{\delta \phi}=\frac{V_{G}}{Q}, \\
\frac{2}{\sigma}\left(\xi \phi^{\prime}-\dot{\phi}\right) & =\frac{\delta S_{0}}{\delta \rho}=Q .
\end{aligned}
$$

It turns out to be convenient to work in the the conformal gauge, i.e. we set $\sigma=1$ and $\xi=0$. Using in addition lightcone variables with $x^{ \pm}=t \pm x$ and squaring (14) yields

$$
4 \partial_{-} \phi \partial_{+} \phi=\left(C-4 \lambda^{2} \phi\right) e^{2 \rho} .
$$

Now using (14) in (15) gives $\dot{\rho} \dot{\phi}=V_{G} / 4$ leading to

$$
\left(\partial_{+}^{2} \phi-2 \partial_{+} \phi \partial_{+} \rho\right)+\left(\partial_{-}^{2} \phi-2 \partial_{-} \phi \partial_{-} \rho\right)=2\left(\lambda^{2} e^{2 \rho}+\partial_{-} \partial_{+} \phi\right),
$$

while $\partial_{-}$and $\partial_{+}$of Eq. (16) gives

$$
\begin{aligned}
-\partial_{-} \phi\left(\lambda^{2} e^{2 \rho}+\partial_{-} \partial_{+} \phi\right) & =\partial_{+} \phi\left(\partial_{-}^{2} \phi-2 \partial_{-} \phi \partial_{-} \rho\right), \\
-\partial_{+} \phi\left(\lambda^{2} e^{2 \rho}+\partial_{-} \partial_{+} \phi\right) & =\partial_{-} \phi\left(\partial_{+}^{2} \phi-2 \partial_{+} \phi \partial_{+} \rho\right) .
\end{aligned}
$$

There are a priori two ways to solve (17), (18), and (19). The first is to set $\partial_{+} \rho=-\partial_{-} \rho$ and $\partial_{+} \phi=-\partial_{-} \phi$, corresponding to static solutions $\dot{\phi}=\dot{\rho}=$ 0 . The second is to assume that the quantities in each of the parenthesis vanishes. This is what we now do; it will become clear that this assumption does not exclude the static solutions. Thus,

$$
\begin{aligned}
& e^{2 \rho} \partial_{-}\left(e^{-2 \rho} \partial_{-} \phi\right)=0 \\
& e^{2 \rho} \partial_{+}\left(e^{-2 \rho} \partial_{+} \phi\right)=0
\end{aligned}
$$

or

$$
\begin{aligned}
& \partial_{-} \phi=e^{2 \rho} A\left(x^{+}\right), \\
& \partial_{+} \phi=e^{2 \rho} B\left(x^{-}\right) .
\end{aligned}
$$

Here, the functions $A\left(x^{+}\right)$and $B\left(x^{-}\right)$are non-vanishing and have opposite signs, but are otherwise arbitrary. Making use of (21), the solution for $\phi$ in (16) may be written

$$
\frac{C}{4}-\lambda^{2} \phi=u e^{-\lambda^{2} \int^{x^{+}} \frac{d x^{+1}}{A\left(x^{+1}\right)}} e^{-\lambda^{2} \int^{x^{-}} \frac{d x^{-1}}{B\left(x^{-1}\right)}},
$$


where $u$ is a (negative) constant. Now inserting (22) and (21) into (16) shows that for given $A\left(x^{+}\right)$and $B\left(x^{-}\right)$, the conformal factor takes the form of a product

$$
e^{2 \rho}=u \frac{e^{-\lambda^{2} \int^{x^{+}} \frac{d x^{+\prime}}{A\left(x^{+\prime}\right)}}}{A\left(x^{+}\right)} \frac{e^{-\lambda^{2} \int^{x^{-}} \frac{d x^{-1}}{B\left(x^{-1}\right)}}}{B\left(x^{-}\right)} .
$$

But within the conformal gauge, a change of coordinates $\tilde{x}^{+}\left(x^{+}\right)$and $\tilde{x}^{-}\left(x^{-}\right)$ can always be made and the conformal factor then undergoes the transformation $e^{2 \rho} \rightarrow e^{2 \rho} \frac{d x^{+}}{d \tilde{x}^{+}} \frac{d x^{-}}{d \tilde{x}^{-}}$. In view of (23), it is clear that the various $A\left(x^{+}\right)$and $B\left(x^{-}\right)$simply correspond to different choices of coordinates. In particular, one can pick the Kruskal gauge by arranging for $\rho$ to vanish. In that case, (20) yields

$$
\phi=a_{1} x^{+} x^{-}+a_{2} x^{+}+a_{3} x^{-}+a_{4},
$$

where $a_{i}$ 's are constants. Inserting this into (16), one finds $a_{1}=-\lambda^{2}$ and $a_{2} a_{3}+\lambda^{2} a_{4}=C / 4$. By an appropriate translation along $x^{+}$and $x^{-}, a_{2}$ and $a_{3}$ can always be set to zero. A general solution is thus

$$
\rho=0 \quad \phi=\frac{C}{4 \lambda^{2}}-\lambda^{2} x^{-} x^{+} .
$$

When reverting back to the initial variables, this is (as expected) the CGHS [8] black hole solution

$$
d s^{2}=-e^{2 \bar{\rho}} d x^{+} d x^{-}
$$

with $\bar{\rho}=\bar{\phi}$ and $e^{-2 \bar{\rho}}=\frac{C}{4 \lambda^{2}}-\lambda^{2} x^{-} x^{+}$, with an ADM mass $M \equiv C / 4 \lambda$. That this quantity is the ADM mass can be understood from an analysis of the Hamiltonian [12]. A special case is the "linear dilaton vacuum solution" (LDV) which is obtained for $C=0$. Clearly, a different choice of coordinates will lead to $A\left(x^{+}\right)=1$ and $B\left(x^{-}\right)=-1$. From (21) one recognizes that these are the static solutions which were obtained in Ref. [15] to prove the validity

of Birkhoff's theorem in two-dimensional dilaton gravity. Evaluating the observable (13) for the CGHS solution derived above just yields the constant $C$.

\section{2 $S_{0}$ and the entropy}

In the following we evaluate Hamilton's principal function (12) on a spacetime that is recovered from the Hamilton-Jacobi equation (11). This is interesting 
because one can discuss a relation to the entropy of the black hole. In the conformal gauge we have with (14) and (15)

$$
S_{0}=\int d x\left[-2 \dot{\phi}+\phi^{\prime} \ln \left(\frac{\phi^{\prime}+\dot{\phi}}{\phi^{\prime}-\dot{\phi}}\right)\right],
$$

which only depends on the dilaton field. For the CGHS solution (25), (27) reads

$$
S_{0}=2 \lambda^{2} \int d x\left[2 t+x \ln \left(\frac{x-t}{x+t}\right)\right]
$$

Interestingly, the real part of (28) is vanishing (contributions from interior and exterior cancel one another separately for $x<0$ and $x>0$ ). The imaginary part has been interpreted in [12 as being proportional to the entropy of the black hole. We note from (27) that

$$
\operatorname{Im} S_{0}=\int_{-\infty}^{\infty} d x \phi^{\prime} \operatorname{Im}\left(\ln \frac{2 \phi^{\prime}-Q}{2 \phi^{\prime}+Q}\right)
$$

Since this yields a non-vanishing value only if $4 \phi^{\prime 2}-Q^{2} \leq 0$, i.e., inside the horizon, one has

$$
\operatorname{Im} S_{0}=\pi \int_{-t}^{t} d x \phi^{\prime}=0
$$

How can this result be reconciled with the non-vanishing entropy of the hole? The contribution to $\operatorname{Im} S_{0}$ from each horizon is

$$
\left.\pi \phi\right|_{\text {horizon }}=\frac{C \pi}{4 \lambda^{2}},
$$

which is one quarter of the hole's entropy. The result in 12 will thus be obtained if only the crossing point of the hypersurface $t=$ constant $>0$ with the upper branch of the future horizon is taken into account?2. All this is independent of the specific hypersurface chosen. However, crossing to the inside of the line of singularity $\phi=0$ should not be done, as it implies an imaginary value for the physical field $\bar{\phi}$. Thus, one should in principle keep $\lambda t<t_{c} \equiv \sqrt{M / \lambda^{3}}$. For $t>t_{c}$, integrating for positive $x$ down to the line of singularity $\phi=0$ would give a single horizon crossing, with now $\operatorname{Im} S_{0}=C \pi / 4 \lambda^{2}$, but of course with a non-vanishing real part.

\footnotetext{
${ }^{2}$ One of us (J.-G. D.) is grateful to G. Kunstatter for discussions about this point.
} 
To get an idea of how general the vanishing of the real part $\operatorname{Re} S_{0}$ is, we consider a different model. For four-dimensional spherically symmetric gravity, where the angular part is frozen, the corresponding two-dimensional dilaton gravity action is similar to (2), but with a modified potential $4 \lambda^{2} \rightarrow$ $1 / \sqrt{2 \phi}$. The associated black hole solution of mass $m$ is 12

$$
\begin{aligned}
\phi & =\frac{1}{2} r^{2} \\
d s^{2} & \left.=r\left[-(1-2 m / r) d t^{2}+(1-2 m / r)^{-1}\right) d r^{2}\right] \\
& =-2 m e^{-\frac{r}{2 m}} d \bar{u} d \bar{v} .
\end{aligned}
$$

In the last equality for the metric, we made use of the usual Kruskal coordinates, $\bar{u}=-4 m e^{-u / 4 m}$ and $\bar{v}=4 m e^{v / 4 m}$, where $u=t-r^{*}$ and $v=t+r^{*}$ and $r^{*}=r+2 m \ln |r / 2 m-1|$ is the usual tortoise coordinate. The expression for $S_{0}$ evaluated on a slice of constant Kruskal time $T$, defined from the null

coordinates through $\bar{u} \bar{v}=T^{2}-X^{2}$, is (we note that [9] gives the solution to the Hamilton-Jacobi equation (11) for arbitrary potentials)

$$
S_{0}=\frac{1}{2} \int d X e^{-\frac{r}{2 m}}\left[2 T+X \ln \left(\frac{X-T}{X+T}\right)\right],
$$

in close resemblance with (28). However, the additional exponential factor makes $\operatorname{Re} S_{0}$ non-vanishing, as can easily be checked numerically.

\section{Matter evolution}

\subsection{Recovery of the Schrödinger Equation}

At the next order, $G^{0}$, we have

$$
-\frac{1}{2}\left(\frac{\delta S_{0}}{\delta \phi} \frac{\delta S_{1}}{\delta \rho}+\frac{\delta S_{0}}{\delta \rho} \frac{\delta S_{1}}{\delta \phi}\right)-\frac{1}{2 i} \frac{\delta^{2} S_{0}}{\delta \rho \delta \phi}+\frac{1}{2}\left(e^{-i S_{1}} \frac{-\delta^{2}\left(e^{i S_{1}}\right)}{\delta f^{2}}+f^{\prime 2}\right)=0 .
$$

We now follow the four-dimensional case [5] and set $e^{i S_{1}}=D^{-1}[\rho, \phi] \chi[\rho, \phi, f]$ so that (34) can be written as

$$
\begin{aligned}
& \frac{-i D^{-1}}{2}\left(\frac{\delta S_{0}}{\delta \phi} \frac{\delta D}{\delta \rho}+\frac{\delta S_{0}}{\delta \rho} \frac{\delta D}{\delta \phi}\right)-\frac{1}{2 i} \frac{\delta^{2} S_{0}}{\delta \rho \delta \phi} \\
& +\chi^{-1} \frac{1}{2}\left[i \frac{\delta S_{0}}{\delta \phi} \frac{\delta \chi}{\delta \rho}+i \frac{\delta S_{0}}{\delta \rho} \frac{\delta \chi}{\delta \phi}-\frac{\delta^{2} \chi}{\delta f^{2}}+{f^{\prime 2}}^{2} \chi\right]=0 .
\end{aligned}
$$


This can be further simplified if $D$ is assumed to obey the equation

$$
\frac{\delta^{2} S_{0}}{\delta \rho \delta \phi} D-\frac{\delta S_{0}}{\delta \phi} \frac{\delta D}{\delta \rho}-\frac{\delta S_{0}}{\delta \rho} \frac{\delta D}{\delta \phi}=0 .
$$

The first term in (36) is formally infinite since it involves functional derivatives at the same point. Some authors have argued that, after suitable regularization, it can be ignored [13, 14] and that one may solve by $D=1$ as if there were no matter. However, as already mentioned above, this can at best be considered ad hoc, but for the purpose of the present paper it is not necessary to resolve this issue.

We are thus left with

$$
-\frac{i}{2}\left(\frac{\delta S_{0}}{\delta \phi} \frac{\delta \chi}{\delta \rho}+\frac{\delta S_{0}}{\delta \rho} \frac{\delta \chi}{\delta \phi}\right)=\mathcal{H}_{m} \chi,
$$

where $\mathcal{H}_{m}$ is the matter Hamiltonian density, $\mathcal{H}_{m} \equiv \frac{1}{2}\left(-\frac{\delta^{2}}{\delta f^{2}}+f^{\prime 2}\right)$. Use of (37) may appeal to either the first or second equalities in (14) and (15). In the next section, we make use of the second equalities to discuss a difficulty in identifying the LHS of (37) as a Tomonaga-Schwinger type time. Here, we derive results for a classical background, making use of the first equalities. In the conformal gauge $(\sigma=1$ and $\xi=0)$, (37) takes the simple form

$$
i\left(\dot{\rho} \frac{\delta \chi}{\delta \rho}+\dot{\phi} \frac{\delta \chi}{\delta \phi}\right)=\mathcal{H}_{m} \chi,
$$

which can be immediately integrated to yield

$$
i \frac{\partial \chi}{\partial t}=H_{m} \chi
$$

with $H_{m} \equiv \int d x \mathcal{H}_{m}$. This is the functional Schrödinger equation for a free scalar field on a flat background. The only information about the gravitational fields occurs in the definition of time through $\rho$ and $\phi$. Note that the use of the original, physical, field variables yields an equation whose form is equivalent to that of (38):

$$
i\left(\dot{\bar{\rho}} \frac{\delta \chi}{\delta \bar{\rho}}+\dot{\bar{\phi}} \frac{\delta \chi}{\delta \bar{\phi}}\right)=\mathcal{H}_{m} \chi
$$

The reason for this formal similarity is of course the conformal coupling of the matter field in two dimensions. Integrating (40) gives again the free evolution (39), but this time on the black hole background (26). 


\subsection{Consistency}

As it has been shown recently [16], replacing the left-hand side of (37) with a functional derivative $d / \delta \tau(x)$ is not consistent, since the object on the lefthand side does not commute for two different space points. In fact, it was found that this commutator on both sides of (37) amounts to the invariance of $\chi$ under spatial diffeomorphisms. In our simple two-dimensional model, this can easily be shown explicitly. Denote the vector fields - one vector field at each space point $x$ - acting on the left-hand side of (37) by

$$
v(x) \equiv-\frac{1}{2}\left(\frac{V_{G}}{Q} \frac{\delta}{\delta \rho}+Q \frac{\delta}{\delta \phi}\right)
$$

and consider their smeared-out version [16]

$$
v^{N} \equiv \int d x N(x) v(x)
$$

Consistency of (37) then demands that

$$
\left[v^{N}, v^{M}\right] \chi=\left[H_{m}^{M}, H_{m}^{N}\right] \chi
$$

where, of course, $H_{m}^{N} \equiv \int d x N(x) \mathcal{H}_{m}(x)$. Explicit calculation yields for the left-hand side

$$
\left[v^{N}, v^{M}\right] \chi=\int d x\left(N M^{\prime}-M N^{\prime}\right)\left(\phi^{\prime} \frac{\delta}{\delta \phi}+\rho^{\prime} \frac{\delta}{\delta \rho}-\frac{d}{d x} \frac{\delta}{\delta \rho}\right) \chi,
$$

while for the right-hand side one finds

$$
\left[H_{m}^{M}, H_{m}^{N}\right] \chi=\int d x\left(M N^{\prime}-N M^{\prime}\right) f^{\prime} \frac{\delta \chi}{\delta f} .
$$

This, however, is nothing but the momentum constraint (5) in this order of approximation. As has been emphasized in [16], equations like (37) have to be properly interpreted in their integrated form, as a functional Schrödinger equation, after a specific choice for lapse and shift has been made, so as to recover quantum field theory on a specific family of spacetimes. This does not, however, mean that the local form of the Schrödinger equation (the Tomonaga-Schwinger equations) is useless, since it may be used for formal considerations, for example the discussion of the correction terms in Section 4.5. 


\subsection{Hawking radiation in a collapsing geometry}

We now show how to recover Hawking radiation from solutions to (39). This is complementary to the standard analysis employing the calculation of Bogolubov coefficients [17] in that the use of the Schrödinger picture brings out interesting new aspects. Rather than an eternal black hole (26), we first consider the 'collapsing' background of 81. The formally analogous case of the eternal black hole (26) will be treated in the next subsection. Although (39) was obtained in the context of the eternal black hole, it is clear from the point of view of quantum field theory on curved geometries and from conformal invariance that it also governs the evolution on an arbitrary twodimensional background, where the variable $t$ stands for the time variable of the conformally flat metric. The collapsing spacetime we are interested in can be obtained from the action (目) by assuming that a left moving shock wave of classical $f$ - matter is imparted (for example) at $\lambda x^{+}=1$, producing a stress tensor $\frac{1}{2} \partial_{+} f \partial_{+} f=\lambda M \delta\left(\lambda x^{+}-1\right)$, so as to form the black hole. In the Kruskal gauge, where $\bar{\rho}=\bar{\phi}$, one then has the background $d s^{2}=-e^{2 \bar{\rho}} d x^{+} d x^{-}$ with

$$
e^{-2 \bar{\rho}}=e^{-2 \bar{\phi}}=\frac{M}{\lambda}\left(1-\lambda x^{+}\right) \Theta\left(\lambda x^{+}-1\right)-\lambda^{2} x^{+} x^{-}
$$

where $\Theta(x)$ is the usual step function. The corresponding Penrose diagram is presented in Figure 1. One might wonder how a nontrivial effect such as Hawking radiation can emerge from (39), since this equation has the form of a free Schrödinger equation. The reason lies in the proper formulation of boundary conditions. The idea is to start from the vacuum state for the scalar field in the absence of a black hole at early times (the "linear dilaton vacuum" region, LDV), let it then evolve according to (39) and compare it with the vacuum solution in the presence of a black hole at late times. The notion "vacuum state" is here defined with respect to "inertial" coordinates, i.e., coordinates which exhibit explicitly the asymptotic flatness of the metric. For (43), such coordinates are respectively

$$
\begin{array}{lll}
\lambda x^{+}=e^{\lambda y^{+}} & \lambda x^{-}=-\frac{M}{\lambda} e^{-\lambda y^{-}} & \text {LDV region } \\
\lambda x^{+}=1+\frac{\lambda}{M} e^{\lambda v^{+}} & \lambda x^{-}=-\frac{M}{\lambda}-e^{-\lambda v^{-}} & \text {black hole region } .
\end{array}
$$

From these lightcone coordinates, timelike and spacelike directions are defined as usual via $y^{ \pm}=t_{y} \pm y$ and $v^{ \pm}=t_{v} \pm v$. Note that $v^{ \pm}$only covers the region above the shock wave, $\lambda x^{+} \geq 1$, and outside the horizon, 


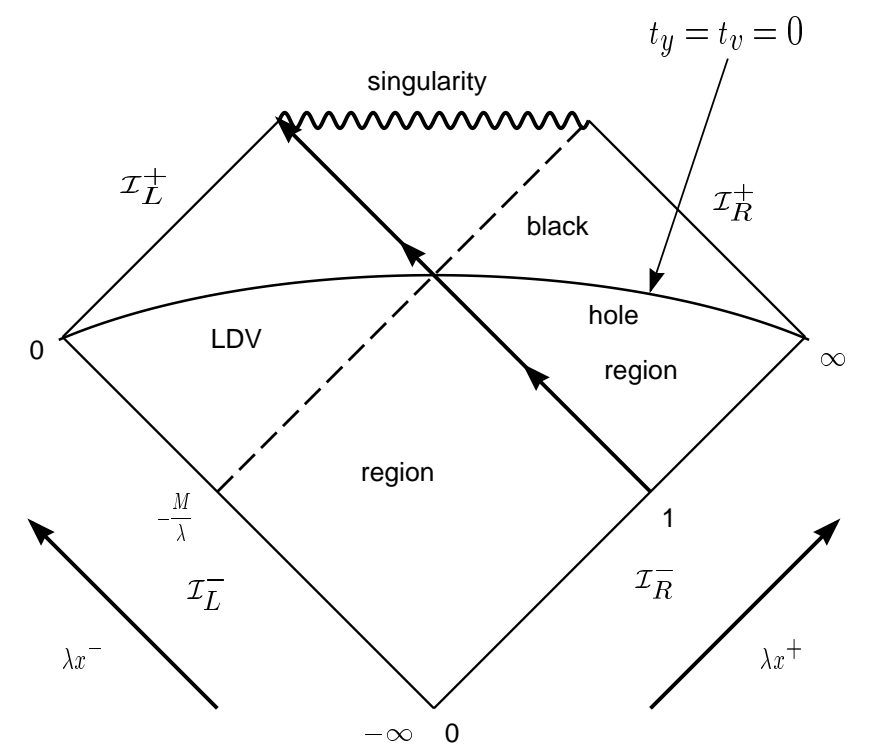

Figure 1: Penrose diagram for the CGHS collapsing black hole. Shock vave (horizon) are indicated by the arrowed (dashed) line. The overlapping slices $t_{y}=t_{v}=0$, where the matter state evolving from the past is compared with the one escaping to $I_{R}^{+}$, are also indicated.

$\lambda x^{-} \lambda \leq-M / \lambda$. The comparison of the vacuum states will be made on the (overlapping) slices $t_{y}=t_{v}=0$. In order to obtain such overlapping slices, the coordinate transformation chosen in (44) is slightly different from a similar one used in the literature, see [1]. As $v \in(-\infty, \infty)$ on the half-line $y \geq 0$, we must demand the boundary condition $f(v) \rightarrow 0$ as $v \rightarrow \pm \infty$ for the field $f$ because otherwise one would not expect the Gaussian functionals which play the role of vacuum states (see below) to converge. In the coordinate $y$ this condition means that $f$ has to vanish at the origin $y=0$. This turns out to be crucial for the following discussion.

It is convenient to consider the Fourier transform of the fields. Because of the boundary condition we have

$$
f(y)=\sqrt{\frac{2}{\pi}} \int_{0}^{\infty} d k \tilde{f}(k) \sin k y
$$

where $\tilde{f}(k)=\tilde{f}^{*}(k)$ (reality of $f(y)$ ). In the following we shall omit the tilde for simplicity and denote the Fourier transform by $f(k)$. It follows from (45) 
that

$$
\frac{\delta}{\delta f(y)}=\sqrt{\frac{2}{\pi}} \int_{0}^{\infty} d k \sin k y \frac{\delta}{\delta f(k)}, \quad \frac{\delta f\left(k^{\prime}\right)}{\delta f(k)}=\delta\left(k-k^{\prime}\right) .
$$

The functional Schrödinger equation (39) then reads

$$
\frac{1}{2} \int_{0}^{\infty} d k\left(-\frac{\delta^{2}}{\delta f^{2}(k)}+k^{2} f^{2}(k)\right) \chi=i \frac{\partial \chi}{\partial t_{y}}
$$

We shall choose the ground state solution of this equation. In the present case this can be represented by a Gaussian functional (see for example [18]), and we have

$$
\chi_{0}\left[f, t_{y}\right)=N \exp \left(-\frac{1}{2} \int_{0}^{\infty} d k k f^{2}(k)-i E_{0} t_{y}\right) .
$$

The ground state energy $E_{0}$ is of course divergent and must be regularized. We shall choose the ground state solution (48) as the initial condition for the Schrödinger equation in the 'future' variables $\left(t_{v}, v\right)$. This equation reads

$$
\frac{1}{2} \int_{-\infty}^{\infty} d v\left(-\frac{\delta^{2}}{\delta f^{2}(v)}+\left[\frac{\partial f}{\partial v}\right]^{2}\right) \chi_{b}=i \frac{\partial \chi_{b}}{\partial t_{v}}
$$

where the subscript "b" refers to the "black hole region". The important difference to above is that $f(v)$ has no restriction at $v=0$. Therefore the Fourier transform is simply given by

$$
f(v)=\int_{-\infty}^{\infty} \frac{d k}{\sqrt{2 \pi}} g(k) e^{i k v}
$$

which has to be contrasted with (45). In that case,

$$
\frac{\delta}{\delta f(v)}=\int_{-\infty}^{\infty} \frac{d k}{\sqrt{2 \pi}} e^{-i k v} \frac{\delta}{\delta g(k)}, \quad \frac{\delta g\left(k^{\prime}\right)}{\delta g(k)}=\delta\left(k-k^{\prime}\right) .
$$

Thus, Eq. (49) becomes

$$
\frac{1}{2} \int_{-\infty}^{\infty} d k\left(-\frac{\delta^{2}}{\delta g(k) \delta g^{*}(k)}+k^{2}|g(k)|^{2}\right) \chi_{b}=i \frac{\partial}{\partial t_{v}} \chi_{b} .
$$


Note that $g(k)$ is complex, whereas $f(k)$ is real. At $t_{y}=t_{v}=0$, the space slices overlap and the states may be compared. The ground state solution to (52) at $t_{v}=0$ reads

$$
\chi_{b, 0}=N \exp \left(-\int_{-\infty}^{\infty} d k|k||g(k)|^{2}\right) .
$$

This is of course different from the previous ground state solution (48). To use (52) we have to rewrite the ground state (48) in terms of the field $g(k)$. We thus write a Bogolubov relation

$$
f(k)=\int_{-\infty}^{\infty} d l \alpha(k, l) g(l) \quad(k>0)
$$

and therefore (using (45) and (50))

$$
\int_{0}^{\infty} d y f(y) \sin k y=\frac{1}{2} \int_{-\infty}^{\infty} d l \alpha(k, l) \int_{-\infty}^{\infty} d v f(v) e^{-i l v} .
$$

One can see by explicit calculation that this can be satisfied by the ansatz

$$
\alpha(k, l)=\frac{1}{\pi} \int_{0}^{\infty} d y \sin k y e^{i l v(y)},
$$

with $v(y)$ being the coordinate transformation (44) at $t_{y}=t_{v}=0$. This leads to

$$
\begin{aligned}
\alpha(k, l) & =\frac{1}{\pi} \int_{0}^{\infty} d y \sin k y e^{\frac{i l}{\lambda} \ln \frac{M}{\lambda}\left(e^{\lambda y}-1\right)} \\
& \approx \frac{1}{\pi}\left(\frac{M}{\lambda}\right)^{\frac{i l}{\lambda}} \int_{0}^{\infty} d y \sin k y(\lambda y)^{\frac{i l}{\lambda}} \\
& \approx \frac{1}{\pi \lambda}\left(\frac{M}{\lambda}\right)^{\frac{i l}{\lambda}} \Gamma\left(1+\frac{i l}{\lambda}\right) \cosh \frac{\pi l}{2 \lambda}\left|\frac{k}{\lambda}\right|^{-1-\frac{i l}{\lambda}} .
\end{aligned}
$$

In the second step of (56), the standard approximation $\left(e^{\lambda y}-1\right) \approx \lambda y$ was made, picking up the dominant contribution in the neighborhood of the horizon [17, 20], and 3.763.1 of [19] was used in the last step. Taking the state (48) at $t_{y}=0$ and expressing it with respect to the field $g(k)$, one finds

$$
\begin{aligned}
\chi_{0} & =N \exp \left(-\frac{1}{2} \int_{0}^{\infty} d k k \int_{-\infty}^{\infty} d p d p^{\prime} \alpha(k, p) \alpha\left(k, p^{\prime}\right) g(p) g\left(p^{\prime}\right)\right) \\
& =N \exp \left(-\int_{-\infty}^{\infty} d p p \operatorname{coth} \frac{\pi p}{2 \lambda}|g(p)|^{2}\right),
\end{aligned}
$$


which is independent of the black hole mass $M$. Because of the analogy to the Rindler case, namely the "loss" of the information on half the space slice behind the horizon, this is in accordance with a similar result found in [21] in the context of accelerated observers and the Unruh radiation.

To solve (52) with the initial condition (57), it is again appropriate to make a Gaussian ansatz:

$$
\chi_{b}=N\left(t_{v}\right) \exp \left(-\int_{-\infty}^{\infty} d p p \Omega\left(p, t_{v}\right)|g(p)|^{2}\right) .
$$

Inserting this into (52) leads to

$$
-\frac{i}{p} \frac{\partial \Omega}{\partial t_{v}}=1-\Omega^{2} .
$$

The solution of this equation with the initial condition $\Omega(p, 0)=\operatorname{coth}(\pi p / 2 \lambda)$ is simply given by

$$
\Omega\left(p, t_{v}\right)=\operatorname{coth}\left(\frac{\pi p}{2 \lambda}+i p t_{v}\right)
$$

We now consider the number operator of the mode with wave number $k$, which is associated with the vacuum state (53) in the presence of the hole. Calculating its expectation value with respect to the state (58) - the timedeveloped state of the "free" vacuum - one finds

$$
\langle n(k)\rangle=\frac{\left(\Omega_{R}-1\right)^{2}+\Omega_{I}^{2}}{4 \Omega_{R}},
$$

where $\Omega_{R}\left(\Omega_{I}\right)$ denotes the real (imaginary) part of $\Omega$. At $t_{v}=0$ this is given by

$$
\langle n(k)\rangle=\frac{1}{e^{\frac{2 \pi|k|}{\lambda}}-1},
$$

i.e. a Planck distribution with the temperature $\lambda / 2 \pi$, as expected. Employing the differential equation (59) one recognizes that $d\langle n\rangle / d t_{v}=0$, i.e. the Planck spectrum is conserved in time.

It is important to emphasize that the Planck spectrum has been recovered from a pure quantum state. This means that there exist other operators than the number operator, from which it is possible to recognize explicitly the difference to a thermal state. Note also that the density matrix of a canonical ensemble of oscillators contains some extra-term besides the cothterm showing up in (57). 


\subsection{Eternal black hole}

Let us now consider how Hawking radiation may arise in the eternal black hole background (26) (which due to its time symmetry would be more properly called a "black-and-white hole"). The derivation in fact closely parallels the one of the collapsing hole, so a brief presentation will suffice. To describe the radiation escaping to the RHS of the black hole, we introduce the coordinates

$$
\begin{aligned}
\lambda t & =e^{\lambda \tilde{x}} \sinh \lambda \tilde{t} \\
\lambda x & =e^{\lambda \tilde{x}} \cosh \lambda \tilde{t},
\end{aligned}
$$

which cover the wedge $|x|>t$ of the manifold. The metric (26) then takes the form $d s^{2}=\left(1+M / \lambda e^{-2 \lambda \tilde{x}}\right)^{-1}\left(-d \tilde{t}^{2}+d \tilde{x}^{2}\right)$ which is thus asymptotically flat far from the black hole. It is clear that the slices $\tilde{t}=0$ and $t=0$ coincide, so that states evolving in $(t, x)$ and $(\tilde{t}, \tilde{x})$ may be compared there. As before, we then have the feature that the range $\tilde{x} \in(-\infty, \infty)$ only covers the half-line $x \geq 0$. As a result, requiring the matter field configuration $f(\tilde{x})$ to vanish at spatial infinity $\tilde{x} \rightarrow \pm \infty$ will translate into $f(x=0)=0$. Note the analogy of (63) to the transformation between Minkowski coordinates and Rindler coordinates, from which it is immediately clear that the Hawking temperature here is $T_{B H}=\lambda / 2 \pi$, independent of the mass. This has recently also been emphasized in [22].

We first determine the vacuum state $\bar{\chi}_{0}[f(k), t]$ which is a solution of the Schrödinger equation (39) in $(t, x)$ coordinates $(f(k)$ being the Fourier tranform of $f(x)$ as in (45). Although the metric is not asymptotically flat in those coordinates, a vacuum state may still be defined. As we do not wish to involve arbitrarily short distance physics, we choose for initial Cauchy

surface a slice $t<0$, but $t>-\sqrt{M / \lambda^{3}}$. Following essentially the same steps as before, $\bar{\chi}_{0}[f(k), t]$ is just the state (48) (with the change $t_{y} \rightarrow t$ ). The state will then evolve up to $t>0$. For the evolution in terms of the $(\tilde{t}, \tilde{x})$ coordinates, the transformation (63) is conformal, so (39) still applies and the corresponding vacuum state at $\tilde{t}=0, \bar{\chi}_{R, 0}[g(k), \tilde{t}=0]$, is thus the same as (53). To determine how $\bar{\chi}_{0}[f(k), t=0]$ reads in terms of $g(k)$, we write a Bogolubov relation as in (54), and find the exact result

$$
\alpha(k, l)=\frac{1}{\pi \lambda} \Gamma\left(1+\frac{i l}{\lambda}\right) \cosh \frac{\pi l}{2 \lambda}\left|\frac{k}{\lambda}\right|^{-1-\frac{i l}{\lambda}} .
$$


Then, following steps analogous to those leading to (62) from (57), we obtain that the state thus evolved according to time $\tilde{t}, \bar{\chi}_{R}[g(k), \tilde{t}]$, has indeed a thermal content in the sense of (62).

We also note that the non-vanishing of $\operatorname{Im} S_{0}$ (compare (29)) is easily understood for the eternal hole. The integral in (29) has to be performed with respect to $\tilde{x}$ which ranges from $-\infty$ to $\infty$ along the half-line originating from the origin in the $(t, x)$-diagram. Since it has only one point in common with the horizon, one directly finds the desired result (31).

\subsection{Corrections to the Schrödinger Equation}

We now proceed to the next order of the semiclassical approximation, $\mathcal{O}(G)$, to show how the functional Schrödinger equation is modified by quantum gravitational corrections [5, 23]. One first obtains an equation involving $S_{2}$,

$$
\begin{aligned}
& -\frac{1}{2}\left(\frac{\delta S_{0}}{\delta \phi} \frac{\delta S_{2}}{\delta \rho}+\rho \leftrightarrow \phi\right)-\frac{1}{2} \frac{\delta S_{1}}{\delta \phi} \frac{\delta S_{1}}{\delta \rho}+\frac{i}{2} \frac{\delta^{2} S_{1}}{\delta \phi \delta \rho} \\
& +\frac{\delta S_{1}}{\delta f} \frac{\delta S_{2}}{\delta f}-\frac{i}{2} \frac{\delta^{2} S_{2}}{\delta f^{2}}=0 .
\end{aligned}
$$

As in the general, four-dimensional, case, this can be greatly simplified by rewriting $S_{2}=\sigma_{2}[\rho, \phi]+\eta[\rho, \phi, f]$ and demanding, in analogy to (36), an equation for $\sigma_{2}$ such that the equations simplify. Since these steps are in full analogy to the general case, we shall be very brief here. Introducing a functional

$$
\psi=\chi e^{i \eta G},
$$

one finds for $\psi$ the "corrected Schrödinger equation"

$$
\begin{aligned}
-\frac{i}{2}\left(\frac{V_{G}}{Q} \frac{\delta \psi}{\delta \rho}+Q \frac{\delta \psi}{\delta \phi}\right) & =\mathcal{H}_{m} \psi+ \\
\frac{G}{\chi}\left(-\frac{1}{2 D} \frac{\delta D}{\delta \rho} \frac{\delta \chi}{\delta \phi}+\phi\right. & \left.\leftrightarrow \rho+\frac{1}{2} \frac{\delta^{2} \chi}{\delta \phi \delta \rho}\right) \psi .
\end{aligned}
$$

If $D=1$, the only correction term reads

$$
\Delta \mathcal{H}_{m} \psi \equiv \frac{G}{2 \chi} \frac{\delta^{2} \chi}{\delta \phi \delta \rho} \psi,
$$


which, of course, involves second order derivatives with respect to the gravitational degrees of freedom. If $\chi$ is a solution to (39), one would expect that it separates in $f$ and the gravitational variables. In this case the correction term would only yield a contribution to the phase, which should not be important. However, as the analysis in [6] suggests, this term should become relevant if the mass of the hole approaches the Planck mass. To evaluate the correction terms properly, one has first to employ a careful regularization, since second functional derivatives are involved. Since this is beyond the scope of this paper, it will be relegated to a future publication.

\section{Decoherence}

The derivation of the functional Schrödinger equations (39) was achieved through the use of a particular WKB state for the gravitational sector. Namely, the choice $\Psi \approx e^{i S_{0}} \chi$ and $\sigma>0$ was made (although the same equation can equally be obtained with $\Psi \approx e^{-i S_{0}} \chi$ and $\left.\sigma<0\right)$. But since the fundamental equations which are assumed here, (8) and (9), are linear, one would, however, expect that arbitrary superpositions of WKB-type states occur. This is suggested also by the real nature of the Wheeler-DeWitt equation, from which it would seem artificial to choose a special complex solution. A more natural state would thus be

$$
\Psi \approx e^{i S_{0}} \bar{\chi}_{R}+e^{-i S_{0}} \bar{\chi}_{R}^{*},
$$

where $\bar{\chi}_{R}$ is the state which evolves out of the eternal black hole geometry and reads

$$
\bar{\chi}_{R}=N(\tilde{t}) \exp \left(-\int_{-\infty}^{\infty} d k k \operatorname{coth}\left(\frac{\pi k}{2 \lambda}+i k \tilde{t}\right)|g(k)|^{2}\right)
$$

The state (69) could naively be called a "superposition of a black hole with a white hole". Such states arise, for example, in the analysis of the gravitational collapse of a dust shell with a sensible boundary condition for the wave function [24]. Another possibility would be

$$
\Psi \approx e^{i S_{0}} \bar{\chi}_{R}+e^{-i S_{0}^{(0)}} \bar{\chi}_{0}
$$


where the second component refers to the linear dilaton vacuum (thus $C=0$ ), i.e.

$$
\bar{\chi}_{0}=N_{0}(\tilde{t}) \exp \left(-\int_{-\infty}^{\infty} d k|k||g(k)|^{2}\right) .
$$

One can interpret the state (71) as a superposition of a black hole with no hole. States of this kind have in fact been discussed extensively in QED and quantum cosmology [25]. It has been demonstrated there that the presence of a huge number of "irrelevant" degrees of freedom may cause decoherence of such states. In the case of QED these may be charged matter states, while in quantum cosmology these may be general matter states or states describing gravitational waves. Because states such as (69) are found in a BornOppenheimer approximation, the decoherence of the various components is nothing but an expression of spontaneous symmetry breaking as it happens, for example, in the case of chiral molecules [26]. What could possibly play the role of the decohering agent in the present case? From the discussion in the preceding section one would expect that Hawking radiation may be able to suppress interferences between the separate WKB-states. This is in particular suggested by its irreversible nature and the fact that it provides a huge entropy capacity.

Writing $\Psi^{*} \Psi$ as a sum of four terms ( $\Psi$ being the superposition (69)), the degree of decoherence between the two semiclassical components can be studied from the following component of the reduced density matrix for the gravitational sector

$$
\rho_{ \pm}[\rho, \phi]=e^{2 i S_{0}} \int \mathcal{D} g \mathcal{D} g^{*} \bar{\chi}_{R}^{2}\left[g, g^{*}, \tilde{t}\right) \equiv e^{2 i S_{0}} D_{ \pm}
$$

and its conjugate $\rho_{\mp}=\rho_{ \pm}^{*}$. Because of the quadratic dependence on the field $g, g^{\dagger}$, the trace in (73) can immediately be evaluated, in full analogy to the QED-case discussed in [25]. One has, with $\Omega(k, \tilde{t})$ given in (60),

$$
\begin{aligned}
D_{ \pm} & =\operatorname{det} \frac{\Omega_{R}}{\Omega}=\exp \left(-\operatorname{Tr} \ln \left(1+i\left(\frac{\Omega_{R}}{\Omega_{I}}\right)\right)\right. \\
& =\exp \left(-i \operatorname{Tr} \frac{\Omega_{I}}{\Omega_{R}}-\frac{1}{2} \operatorname{Tr}\left(\frac{\Omega_{I}}{\Omega_{R}}\right)^{2}-\ldots\right) .
\end{aligned}
$$

The decoherence factor can be be evaluated by noting that the real and 
imaginary parts of $\Omega$ are given, respectively, by the expressions

$$
\begin{aligned}
\Omega_{R} & =-\frac{\sinh \frac{\pi k}{\lambda}}{\cos 2 k \tilde{t}-\cosh \frac{\pi k}{\lambda}} \\
\Omega_{I} & =\frac{\sin 2 k \tilde{t}}{\cos 2 k \tilde{t}-\cosh \frac{\pi k}{\lambda}}
\end{aligned}
$$

which yields

$$
\Omega_{I}=-\frac{\sin 2 k \tilde{t}}{\sinh \frac{\pi k}{\lambda}} \Omega_{R}
$$

One thus has for the exact decoherence factor the expression

$$
D_{ \pm}=\exp \left(-L \int_{0}^{\infty} \frac{d k}{2 \pi} \ln \left[1-i \frac{\sin 2 k \tilde{t}}{\sinh \frac{\pi k}{\lambda}}\right]\right)
$$

where $\operatorname{Tr} \rightarrow L \int d k / 2 \pi$ was used, and $L$ is a regularization length. The important part for the amount of decoherence is its absolute value,

$$
\left|D_{ \pm}\right|=\exp \left(-L \int_{0}^{\infty} \frac{d k}{4 \pi} \ln \left[1+\frac{\sin ^{2} 2 k \tilde{t}}{\sinh ^{2} \frac{\pi k}{\lambda}}\right]\right) .
$$

Since this cannot be exactly evaluated, the expansion of the exponent made in (74) appears appropriate. Considering the first real term in the exponent, one finds

$$
\begin{aligned}
D_{ \pm}^{(1)} & \equiv \exp \left(-\frac{1}{2} \operatorname{Tr}\left(\frac{\Omega_{I}}{\Omega_{R}}\right)^{2}\right)=\exp \left(-\frac{L}{2 \pi} \int_{0}^{\infty} d k \frac{\sin ^{2} 2 k \tilde{t}}{\sinh ^{2} \frac{\pi k}{\lambda}}\right) \\
& =\exp \left(-\frac{L \lambda}{4 \pi^{2}}[2 \lambda \tilde{t} \operatorname{coth} 2 \lambda \tilde{t}-1]\right)
\end{aligned}
$$

where 3.986.4 in 19 was used in the last step. A finite value for $L$ is obtained, if the black hole states are put into a box of finite length, as is frequently done in gedankenexperiments. In the limit $\tilde{t} \rightarrow 0$ this goes to one as it must, since there we matched our state to the one for the dilaton vacuum which has $\Omega_{I}=0$. In the limit $\tilde{t} \rightarrow \infty$ this would yield the simple expression $\exp \left(-L \lambda^{2} \tilde{t} / 2 \pi^{2}\right)$. One must, however, note that higher order terms in the expansion (74) become important in this limit and one has to resort to the 


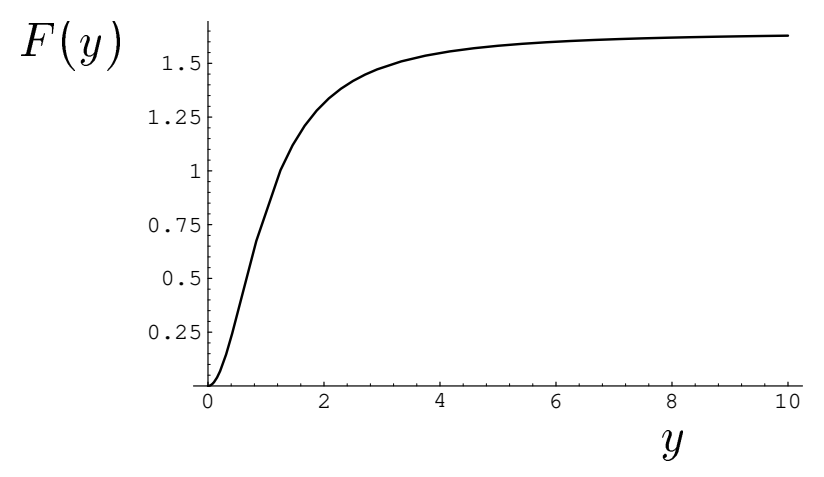

Figure 2: Plot of $F(y)$, where $y \equiv 2 l \tilde{t} / \pi$. After a transitory period of order $l^{-1}$, the decoherence in Eq. (80) reaches a plateau in time $\tilde{t}$.

exact expression (78). Differentiating (78) with respect to $\tilde{t}$ yields an expression which vanishes (due to Riemann's lemma) in the limit of large $\tilde{t}$. The decoherence factor $(78)$ itself thus approaches a constant. It is convenient to write

$$
\ln \left|D_{ \pm}\right|=-\frac{L \lambda}{4 \pi^{2}} F(y)
$$

where $y \equiv 2 \lambda \tilde{t} / \pi$. Simple numerical analysis yields a limiting value for $F$ of about 1.65 which is rapidly obtained for $y$ bigger than about 10, as depicted in Figure 2. Decoherence thus becomes effective for $L \lambda>1$. This can be physically interpreted as follows. The dominating mode for the Hawking temperature $T=\lambda / 2 \pi$ has a wavelength of the order $\lambda^{-1}$. If the black hole is put into a box with radius $L$, interferences are important if $L$ is of the same order than this wavelength, but they become suppressed if the box is larger. One can now calculate the analogous expression for the superposition (71) of a black hole with no hole. Instead of (73) one now has to calculate

$$
\tilde{\rho}_{ \pm}[\rho, \phi]=e^{i S_{0}^{(1)}-i S_{0}^{(0)}} \int \mathcal{D} g \mathcal{D} g^{*} \bar{\chi}_{R} \bar{\chi}_{0}^{*} \equiv e^{i S_{0}^{(1)}-i S_{0}^{(0)}} \tilde{D}_{ \pm},
$$

Inserting the Gaussian functionals $\bar{\chi}_{R}$ and $\bar{\chi}_{0}$ into this expressions, one recognizes that now $\tilde{D}_{ \pm}=\operatorname{det}\left[\tilde{\Omega}_{R} /\left(\tilde{\Omega}_{R}+i \tilde{\Omega}_{I}\right)\right]$, where $\tilde{\Omega}_{R}=\Omega_{R}+1$ and $\tilde{\Omega}_{I}=\Omega_{I}$ with $\Omega_{R}$ and $\Omega_{I}$ given by the expressions (75). In this example one then finds

$$
\frac{\tilde{\Omega}_{I}}{\tilde{\Omega}_{R}}=-\frac{\sin 2 k \tilde{t}}{e^{\frac{\pi k}{\lambda}}-\cos 2 k \tilde{t}} .
$$


This then leads instead of (80) to an expression for the decoherence factor of the form

$$
\ln \left|\tilde{D}_{ \pm}\right|=-\frac{L \lambda}{4 \pi^{2}} \tilde{F}(y)
$$

where

$$
\tilde{F}(y)=\int_{0}^{\infty} d x \ln \left(1+\frac{\sin ^{2} x y}{\left(e^{x}-\cos x y\right)^{2}}\right)<F(y) .
$$

One has $\tilde{F}<F$ for all $y$ because only one component in (71) carries Hawking radiation. Again, $\tilde{F}(y)$ approaches a constant for large $y$, but - in contrast to $F$ - it is not monotonic. A numerical evaluation yields, for example, the value $\tilde{F}(15) \approx 0.413$ (compared to $F(15) \approx 1.637$ ).

Since the state $\bar{\chi}_{R}$ does not depend on the black hole mass, there is of course no decoherence for a superposition corresponding to different masses, in contrast to the case of accelerating detectors [28]. This is, however, a peculiarity of the two-dimensional model. In four dimensions, the Hawking temperature is inversely proportional to the mass, so one would expect decoherence for not-to-large masses. Heuristically, one can take this into account by replacing $\lambda$ in the above expressions for the states by $(4 G M)^{-1}$. One would thus expect the decoherence factor in four spacetime dimensions to be of the limiting form for large times

$$
D_{ \pm} \approx \exp \left(- \text { constant } \times\left(\frac{L}{4 G M}\right)^{3}\right) .
$$

This expression depends now explicitly on the mass of the hole, and one recognizes that decoherence is efficient for small masses where the Hawking radiation is large. Again, decoherence for a black hole in a box of dimensions $L^{3}$ would be efficient for the realistic case of the length $L$ being much bigger than the Schwarzschild radius.

\section{Conclusions}

The central issue in our paper is the investigation of the semiclassical approximation in the context of two-dimensional dilaton gravity. We have demonstrated how Hawking radiation can be properly understood in the 
Schrödinger representation and how the validity of the semiclassical approximation can be investigated. We have shown, in particular, how the correlation between the Hawking radiation and the black hole can decohere the latter - the various semiclassical components become dynamically independent.

We want to conclude in mentioning some of the interesting open problems which are topics for future research. Hawking radiation was obtained in our framework from a pure quantum state outside the horizon. While the expectation value of the number operator for the field modes exhibits a perfect Planckian spectrum, there are of course higher order operators which distinguish this state from a thermal state. The occurrence of such a pure state can be understood from the analogy with the Rindler case, where boundary conditions corresponding to the presence of a mirror at the origin can be posed. Alternatively, one can consider a quantum state on the complete manifold [21]. Tracing out the degrees of freedom of the left wedge would then lead to the well-known thermal density matrix in the right wedge. The derivation of the decoherence factor would proceed along the lines described in Section (5).

Another important problem is the possible occurrence of anomalies which could spoil the whole semiclassical limit [10]. This of course requires a full understanding of regularization in quantum gravity. A proper regularization is also needed for a rigorous evaluation of the correction terms to the Schrödinger equation, which have been derived in Section 4.5. Maybe one can make use of the methods developed in [27] and [29] where the attempt is made, in the context of a strong coupling expansion, to define the kinetic terms rigorously. One can also try to modify the expansion scheme itself [30].

We also wish to comment briefly how our results are related to those presented in [7]. There, a vacuum matter state was freely evolved on the geometry of Figure 1 for a black hole of mass $M$ and then compared, through an appropriate scalar product, to the same evolution on a black hole of mass $M+\Delta M$. It was claimed there that a value of $\Delta M / \lambda$ of the order of $e^{-M / \lambda}$ would lead to a vanishing product, signalling a breakdown of the semiclassical approximation (if $\lambda$ is taken to be proportional to the inverse Planck length). First we note that considering a scalar product is essentially what was done in Section 5, although there we have not considered superpositions with different masses. But since the matter states describing Hawking radiation are identical for two different black hole masses, we would reach the 
opposite conclusion of an absence rather than an excess of decoherence for superpositions of different masses. It should be remembered, however, that the foliation chosen is quite different. In our case, before reaching the horizon, our spacelike slices extend to the left spatial infinity, while for times after the shock wave, the matter evolution has support on the coordinate system $v^{ \pm}$which only covers the region outside the horizon (analogous to the Unruh effect). In this way, the aspect of information for half the line being 'lost' behind the horizon is captured. In [7], the matter states are compared on slices that cross the shock wave and the horizon. Given the different slicing studied, further work would be needed to clarify how the two approaches are related. From a practical point of view, we note the relative simplicity of our calculation which makes use of the Schrödinger picture througout.

\section{ACKNOWLEDGMENTS}

We would like to thank Robert Myers for useful comments on the manuscript. 


\section{References}

[1] A. Strominger, "Les Houches Lectures on Black Holes", hep-th/9501071.

[2] K. V. Kuchař, Phys. Rev. D50 (1994) 3961.

[3] H. A. Kastrup and T. Thiemann, Nucl. Phys. B 425 (1994) 665.

[4] J. D. Romano, "Spherical Symmetric Scalar Field Collapse: An example of the Spacetime Problem of Time", gr-qc/9501015.

[5] C. Kiefer, in Canonical Gravity: From classical to quantum, edited by J. Ehlers and H. Friedrich (Springer - Verlag, Berlin, 1994).

[6] C. Kiefer, R. Müller, and T. P. Singh, Mod. Phys. Lett. A9 (1994) 2661.

[7] E. Keski-Vakkuri, G. Lifschytz, S. D. Mathur, and M. E. Ortiz, Phys. Rev. D51 (1995) 1764.

[8] C.G. Callan, S.B. Giddings, J.A. Harvey, and A. Strominger, Phys. Rev. D45 (1992) R1005.

[9] D. Louis-Martinez, J. Gegenberg, and G. Kunstatter, Phys. Lett. 321B (1994) 193.

[10] D. Cangemi, R. Jackiw, and B. Zwiebach, "Physical States in MatterCoupled Dilaton Gravity", to appear in Annals of Physics.

[11] S. P. de Alwis and D. A. MacIntire, Phys. Rev. D50 (1994) 5164.

[12] J. Gegenberg, G. Kunstatter, and D. Louis-Martinez, Phys. Rev. D51 (1995) 1781.

[13] T. Hori, Prog. Theor. Phys. 90 (1993) 743.

[14] B. DeWitt, Phys. Rev. 160 (1967) 1113.

[15] D. Louis-Martinez and G. Kunstatter, Phys. Rev. D49 (1994) 5227.

[16] D. Giulini and C. Kiefer, Class. Quantum Grav. 12 (1995) 403.

[17] S. Giddings and W. Nelson, Phys. Rev. D46 (1992) 2486. 
[18] R. Jackiw, in Field Theory and Particle Physics, edited by O. Eboli, M. Gomes, and A. Santano (World Scientific, Singapore, 1988).

[19] I. S. Gradshteyn and I. M. Ryzhik, Table of Integrals, Series, and Products (Academic Press, Orlando, 1980).

[20] S. W. Hawking, Commun. Math. Phys. 43 (1975) 199.

[21] K. Freese, C. T. Hill, and M. Mueller, Nucl. Phys. B255 (1985) 693.

[22] M. Cadoni and S. Mignemi, Phys. Lett. 358B (1995) 217.

[23] C. Kiefer and T. P. Singh, Phys. Rev. D44 (1991) 1067.

[24] P. Hájíček, Commun. Math. Phys. 150 (1992) 545.

[25] C. Kiefer, Phys. Rev. D46 (1992) 1658.

[26] H. D. Zeh, in Stochastic evolution of quantum states in open systems and measurement processes, edited by L. Diósi and B. Lukács (World Scientific, Singapore, 1994).

[27] T. Horiguchi, K. Maeda, and M. Sakamoto, Phys. Lett. 344B (1995) 105.

[28] J.-G. Demers, Mod. Phys. Lett. A10 (1995) 1745.

[29] P. Mansfield, Nucl. Phys. B148 (1994) 1130.

[30] S. P. Kim, Phys. Rev. D52 (1995) 3382. 